\title{
CONTROLLABILITY OF NONLINEAR IMPLICIT FRACTIONAL INTEGRODIFFERENTIAL SYSTEMS
}

\author{
KRISHNAN BALACHANDRAN, SHANMUGAM DIVYA \\ Department of Mathematics \\ Bharathiar University, Coimbatore 641 046, India \\ e-mail: \{kb.maths.bu, divya.mathsbu\} @gmail.com
}

\begin{abstract}
In this paper, we study the controllability of nonlinear fractional integrodifferential systems with implicit fractional derivative. Sufficient conditions for controllability results are obtained through the notion of the measure of noncompactness of a set and Darbo's fixed point theorem. Examples are included to verify the result.
\end{abstract}

Keywords: controllability, fractional derivative, integrodifferential equations, fixed point theorem.

\section{Introduction}

Integrodifferential equations arise in many fields of science and engineering such as fluid dynamics, biological models, and chemical kinetics. A detailed investigation of integrodifferential equations and their solution via the Laplace transform method can be found in the work of Burton (1983). Recently, fractional integrodifferential equations have been used to model various physical phenomena such as heat conduction in materials with memory, combined conduction, convection and radiation problems (Caputo, 1967; Olmstead and Handelsman, 1976; Sabatier et al., 2007), and numerical methods for such equations can be found in the works of Mittal and Nigam (2008) as well as Rawashdeh (2011). Models represented by neutral differential equations are encountered in theoretical epidemiology, physiology and population dynamics. It is interesting to introduce a fractional derivative for these models and study their qualitative behaviors.

Controllability is one of the fundamental concepts in control theory and plays a major role in many control problems such as stabilization of unstable systems by feedback or optimal control (Klamka, 1993). This problem can be studied by using different techniques, among which the fixed-point technique is the most powerful method for establishing the controllability results of nonlinear dynamical systems (see Balachandran and Dauer, 1987; Klamka, 1975a; 1975b; 1975c; 1999; 2001; 2008). Dacka (1980) introduced a method based on the measure of non compactness of a set and Darbo's fixed-point theorem for studying the controllability of nonlinear systems with an implicit derivative. This method was extended to a larger class of dynamical systems by Balachandran (1988).

Anichini et al. (1986) addressed the controllability problem for nonlinear systems through the notion of the measure of noncompactness, the condensing operator and the Sadovskii fixed point theorem (Sadovskii, 1972), whereas Balachandran and Balasubramaniam (1992; 1994) considered the same problem for nonlinear Volterra integrodifferential systems with an implicit derivative. Klamka (2010) discussed the minimum energy control problem of infinite-dimensional fractional-discrete time linear systems and established necessary and sufficient conditions for exact controllability of such systems. Recently, Balachandran et al. (2012a; 2013a; 2013b; 2012b; 2012c; 2012d) studied the controllability problem for various types of nonlinear fractional dynamical systems by using fixed point theorems.

However, no work has been reported on the controllability of nonlinear implicit fractional integrodifferential systems in the literature. Therefore, in this paper we study the controllability of nonlinear implicit fractional integrodifferential systems and neutral fractional integrodifferential systems by using the measure of non compactness of a set and the Darbo fixed-point theorem. 


\section{Preliminaries}

In this section we give some basic definitions and properties of fractional operators, the special function and the solution representation of fractional integrodifferential equations (Kexue and Jigen, 2011; Kilbas et al., 2006; Miller and Ross, 1993; Oldham and Spanier, 1974; Podlubny, 1999; Samko et al., 1993; Kaczorek, 2011).

For $\alpha, \beta>0$, with $n-1<\alpha<n, n-1<\beta<$ $n$, and $n \in \mathbb{N}, D$ is the usual differential operator and suppose that $f \in L_{1}\left(\mathbb{R}_{+}\right), \mathbb{R}_{+}=[0, \infty)$.

The Riemann-Liouville fractional integral operator is defined as

$$
\begin{aligned}
\left(I_{0+}^{\alpha} f\right)(t) & =\frac{1}{\Gamma(\alpha)} \int_{0}^{t}(t-s)^{\alpha-1} f(s) \mathrm{d} s, \\
\left(D_{0+}^{\alpha} f\right)(t) & =D^{n}\left(I_{0+}^{n-\alpha} f\right)(t),
\end{aligned}
$$

and the Caputo fractional derivative is taken as

$$
{ }^{C} D_{0+}^{\alpha} f(t)=\left(I_{0+}^{n-\alpha} D^{n} f\right)(t), \quad 0<\alpha<1,
$$

and, in particular, $I_{0+}^{\alpha}{ }^{C} D_{0+}^{\alpha} f(t)=f(t)-f(0)$

The following is a well-known relation for the Riemann-Liouville and the Caputo derivative:

$$
\begin{array}{r}
{ }^{C} D_{0+}^{\alpha} f(t)=D_{0+}^{\alpha} f(t)-\sum_{k=0}^{n-1} \frac{t^{k-\alpha}}{\Gamma(k-\alpha+1)} f^{(k)}\left(0^{+}\right), \\
n=\Re(\alpha)+1 .
\end{array}
$$

An interesting class of functions introduced by Mittag-Leffler is

$$
E_{\alpha, \beta}(z)=\sum_{k=0}^{\infty} \frac{z^{k}}{\Gamma(\alpha k+\beta)} \quad \text { for } \alpha, \beta>0, \quad z \in \mathbb{C}
$$

and, in particular, for $\beta=1$,

$$
E_{\alpha, 1}\left(a z^{\alpha}\right)=E_{\alpha}\left(a z^{\alpha}\right)=\sum_{k=0}^{\infty} \frac{a^{k} z^{\alpha k}}{\Gamma(\alpha k+1)}, \quad a, z \in \mathbb{C} .
$$

Further, the Laplace transform of the Caputo fractional derivative and the Mittag-Leffler function are

(i) $\mathcal{L}\left\{{ }^{C} D_{0+}^{\alpha} f(t)\right\}(s)=s^{\alpha} F(s)-\sum_{k=0}^{n-1} f^{k}\left(0^{+}\right) s^{\alpha-k-1}$,

(ii) $\mathcal{L}\left\{t^{\beta-1} E_{\alpha, \beta}\left( \pm a t^{\alpha}\right)\right\}(s)=\frac{s^{\alpha-\beta}}{s^{\alpha} \mp a}$ for $\Re(s)>|a|^{\frac{1}{\alpha}}$ and $\Re(\beta)>0$,

(iii) $\mathcal{L}\left\{E_{\alpha}\left( \pm a t^{\alpha}\right)\right\}(s)=\frac{s^{\alpha-1}}{s^{\alpha} \mp a}$ for $\beta=1$.
For brevity, let us take $I_{0+}^{\alpha}$ as $I^{\alpha}$ and ${ }^{C} D_{0+}^{\alpha}$ as ${ }^{C} D^{\alpha}$.

Consider the linear fractional integrodifferential equation of the form

$$
\begin{aligned}
{ }^{C} D^{\alpha} x(t) & =A x(t)+\int_{0}^{t} H(t-s) x(s) \mathrm{d} s, \\
x(0) & =x_{0}
\end{aligned}
$$

where $t \in[0, T]:=J, 0<\alpha<1, x(t) \in \mathbb{R}^{n}, A$ is an $n \times$ $n$ matrix and $H$ is an $n \times n$ continuous matrix. Taking the Laplace transform on both the sides of the above equation and using the Laplace transform of the Caputo derivative, we get

$$
\begin{aligned}
s^{\alpha} X(s)-s^{\alpha-1} x(0) & =A X(s)+H(s) X(s), \\
X(s) & =s^{\alpha-1}\left(s^{\alpha} I-A-H(s)\right)^{-1} x_{0} .
\end{aligned}
$$

Taking the inverse Laplace transform on both the sides of the above equation, we have

$$
\begin{aligned}
& \mathcal{L}^{-1}\{X(s)\}(t) \\
& =\mathcal{L}^{-1}\left\{s^{\alpha-1}\left(s^{\alpha} I-A-H(s)\right)^{-1}\right\}(t) x_{0}, \\
x(t) & =R_{\alpha}(t) x_{0},
\end{aligned}
$$

where $R_{\alpha}(t)$ is an $n \times n$ matrix satisfying the following conditions:

(a) $R_{\alpha}(0)=I$,

(b) ${ }^{C} D^{\alpha} R_{\alpha}(t)=A R_{\alpha}(t)+\int_{0}^{t} H(t-s) R_{\alpha}(s) \mathrm{d} s$,

(c) $\mathcal{L}\left\{R_{\alpha}(t)\right\}(s)=\int_{0}^{t} e^{-s t} R_{\alpha}(t) \mathrm{d} t:=s^{\alpha-1}\left(s^{\alpha} I-A-\right.$ $H(s))^{-1}$.

Consider the linear fractional dynamical system represented by the following fractional integrodifferential equation:

$$
\left\{\begin{aligned}
{ }^{C} D^{\alpha} x(t)= & A x(t)+\int_{0}^{t} H(t-s) x(s) \mathrm{d} s \\
& +B u(t), \quad t \in J, \quad 0<\alpha<1, \\
x(0)= & x_{0},
\end{aligned}\right.
$$

where $A, B$ are $n \times n, n \times m$ matrices, respectively, $x(t) \in$ $\mathbb{R}^{n}$ and $u(t) \in \mathbb{R}^{m}$ are the state and control vectors of the system and $H$ is an $n \times n$ continuous matrix. The solution of the system (2) is given by

$$
x(t)=R_{\alpha}(t)+\int_{0}^{t}(t-s)^{\alpha-1} R_{\alpha, \alpha}(t-s) B u(s) \mathrm{d} s,
$$

where

$$
R_{\alpha, \alpha}(\theta)=\theta^{1-\alpha} \frac{\mathrm{d}}{\mathrm{d} \theta}\left(\int_{0}^{t} \frac{(\theta-\tau)^{\alpha-1}}{\Gamma(\alpha)} R_{\alpha}(\tau) \mathrm{d} \tau\right) .
$$

Definition 1. The system (2) is said to be controllable on $J$ if, for every $x_{0}, x_{1} \in \mathbb{R}^{n}$, there exists a control $u(t)$ 
such that the solution $x(t)$ of the system (2) satisfies the condition $x(0)=x_{0}$ and $x(T)=x_{1}$.

Define the controllability Grammian matrix $G$ as

$$
\begin{aligned}
G & \int_{0}^{T}(T-s)^{\alpha-1}\left[R_{\alpha, \alpha}(T-s) B\right]\left[R_{\alpha, \alpha}(T-s) B\right]^{*} \mathrm{~d} s,
\end{aligned}
$$

where $*$ denotes the matrix transpose. It is proved that the linear system (2) is controllable on $J$ if and only if the controllability Grammian matrix $G$ is positive definite for some $T>0$ (Balachandran and Kokila, 2013a).

\section{Fractional integrodifferential systems}

In this section we consider the fractional system represented by the fractional integrodifferential equation with an implicit fractional derivative of the form

$$
\begin{aligned}
{ }^{C} D^{\alpha} x(t)= & A x(t)+\int_{0}^{t} H(t-s) x(s) \mathrm{d} s+B u(t) \\
& +f\left(t, x(t),{ }^{C} D^{\alpha} x(t), u(t)\right), \\
x(0)= & x_{0}
\end{aligned}
$$

where $0<\alpha<1, t \in J, x \in \mathbb{R}^{n}, u \in \mathbb{R}^{m}, A, B$ are respectively $n \times n, n \times m$ matrices, $H$ is an $n \times n$ continuous matrix and the nonlinear function $f: J \times \mathbb{R}^{n} \times$ $\mathbb{R}^{n} \times \mathbb{R}^{m} \rightarrow \mathbb{R}^{n}$ is continuous.

In order to study this problem, we need some basic facts about the measure of noncompactness and the related fixed-point theorem due to Darbo.

Definition 2. Let $(X,\|\cdot\|)$ be a Banach space and $S$ be a bounded subset of $X$. Then the measure of noncompactness of a set $S$ is defined by $\mu(S)=\inf \{r>0 ; S$ can be covered by a finite number of balls whose radii are smaller than $r\}$.

Let us adopt the following settings:

- Let $C_{n}(J)$ be the space of continuous functions with the norm $\|x\|=\max \left\{x_{i}(t): i=1,2, \ldots, n, t \in\right.$ $J\}$. Then the measure of noncompactness of a bounded subset $E$ in $X$ is given by

$$
\mu(E)=\frac{1}{2} \theta_{0}(E)=\frac{1}{2} \lim _{h \rightarrow 0^{+}} \theta(E, h),
$$

where

$$
\theta(E, h)=\sup _{x \in E}\{\sup |x(t)-x(s)|:|t-s| \leq h\}
$$

is the common modulus of the continuity of the functions which belong to the set $E$.
- We may proceed in a similar way in the case where the space considered is the space $C_{n}^{\alpha}(J)$ with the norm

$$
\|x\|_{C_{n}^{\alpha}}=\|x\|_{C_{n}}+\left\|{ }^{C} D^{\alpha} x\right\|_{C_{n}} .
$$

Then the measure of the noncompactness of a set $E$ is given by

$$
\mu(E)=\frac{1}{2} \theta_{0}\left({ }^{C} D^{\alpha} E\right),
$$

where

$$
{ }^{C} D^{\alpha} E=\left\{{ }^{C} D^{\alpha} x ; x \in E\right\} .
$$

- Set the space of Cartesian product $C_{n+m}^{\alpha}(J)=$ $C_{n}^{\alpha}(J) \times C_{m}(J)$ with the norm

$$
\|(x, u)\|_{C_{n+m}^{\alpha}}=\max \left\{\|x\|_{C_{n}^{\alpha}},\|u\|_{C_{m}}\right\} .
$$

Then the measure of noncompactness of any bounded set $E$ in $C_{n+m}^{\alpha}(J)$ is given by the relation

$$
\mu(E)=\max \left[\mu\left(E_{1}\right), \mu\left(E_{2}\right)\right],
$$

where $E_{1}, E_{2}$ denote the natural projections of the set $E$ on the spaces $C_{n}^{\alpha}(J)$ and $C_{m}(J)$, respectively.

Assume that there exist constants $K>0, k>0$ such that

$$
\left\{\begin{array}{l}
|f(t, x, y, u)| \leq K, \\
|f(t, x, y, u)-f(t, x, \bar{y}, u)| \leq k|y-\bar{y}|,
\end{array}\right.
$$

for all $x, y, \bar{y} \in \mathbb{R}^{n}, u \in \mathbb{R}^{m}$, and $t \in J$.

The following version of Darbo's fixed point theorem, being a generalization of the Schauder fixed-point theorem, shows the usefulness of the measure of noncompactness.

Theorem 1. (Darbo's theorem (Dacka, 1980)). If $M$ is a nonempty bounded closed convex subset of $X$ and $P$ : $M \rightarrow M$ is a continuous mapping such that for any set $E \subset M$ we have $\mu(P E) \leq k \mu(E)$ where $k$ is a constant $0 \leq k<1$, then $P$ has a fixed point.

For each fixed point $(z, v) \in C_{n+m}^{\alpha}(J)$, consider the fractional integrodifferential system of the form

$$
\begin{aligned}
{ }^{C} D^{\alpha} x(t)= & A x(t)+\int_{0}^{t} H(t-s) x(s) \mathrm{d} s+B u(t) \\
& +f\left(t, z(t),{ }^{C} D^{\alpha} z(t), v(t)\right) .
\end{aligned}
$$

The solution of the above system with $x(0)=x_{0}$ can be written as (Balachandran and Kokila, 2013a)

$$
\begin{aligned}
x(t)= & R_{\alpha}(t) x_{0} \\
& +\int_{0}^{t}(t-s)^{\alpha-1} R_{\alpha, \alpha}(t-s) B u(s) \mathrm{d} s \\
& +\int_{0}^{t}(t-s)^{\alpha-1} R_{\alpha, \alpha}(t-s) \\
& \times f\left(s, z(s),{ }^{C} D^{\alpha} z(s), v(s)\right) \mathrm{d} s .
\end{aligned}
$$


Now we prove the main result of the paper.

Theorem 2. If the linear system (2) is controllable on $J$ and the function $f$ satisfies the condition (8), then the nonlinear system (9) is controllable on J.

Proof. Define the operator $\Psi: C_{n+m}^{\alpha}(J) \rightarrow C_{n+m}^{\alpha}(J)$ as in the work of Balachandran and Kokila (2013b) by

$$
\Psi(z, v)=(x, u)
$$

where

$$
\begin{aligned}
u(t)= & B^{*} R_{\alpha, \alpha}^{*}(T-t) G^{-1}\left[x_{1}-R_{\alpha}(T) x_{0}\right. \\
& -\int_{0}^{T}(T-s)^{\alpha-1} R_{\alpha, \alpha}(T-s) \\
& \left.\times f\left(s, z(s),{ }^{C} D^{\alpha} z(s), v(s)\right) \mathrm{d} s\right], \\
x(t)= & R_{\alpha}(t) x_{0}+\int_{0}^{t}(t-s)^{\alpha-1} R_{\alpha, \alpha}(t-s) B \\
& \times B^{*} R_{\alpha, \alpha}^{*}(T-s) G^{-1}\left[x_{1}-R_{\alpha}(T) x_{0}\right. \\
& -\int_{0}^{T} T^{\alpha-1} R_{\alpha, \alpha}(T-\tau) \\
& \left.\times f\left(\tau, z(\tau),{ }^{C} D^{\alpha} z(\tau), v(\tau)\right) \mathrm{d} \tau\right] \mathrm{d} s \\
& +\int_{0}^{t}(t-s)^{\alpha-1} R_{\alpha, \alpha}(t-s) \\
& \times f\left(s, z(s),{ }^{C} D^{\alpha} z(s), v(s)\right) \mathrm{d} s .
\end{aligned}
$$

It can be easily verified that $x(T)=x_{1}$ by substituting $t=$ $T$ in (12). Now we consider the right-hand side of (11) and (12) as a pair of operators $\Psi_{2}([z, v])(t)$ and $\Psi_{1}([z, v])(t)$, respectively, and define the operator $\Psi: C_{n+m}^{\alpha}(J) \rightarrow$ $C_{n+m}^{\alpha}(J)$ by

$$
\Psi([z, v])(t)=\left[\Psi_{1}([z, v])(t), \Psi_{2}([z, v])(t)\right] .
$$

Since all the functions involved in the definition of the operator $\Psi$ are continuous, $\Psi$ is continuous. To prove that $\Psi$ maps the space $C_{n+m}^{\alpha}(J)$ into itself, consider the closed convex set $E$ of $C_{n+m}^{\alpha}(J)$ defined by

$$
E=\left\{[z, v] ;\|v\| \leq N_{1},\|z\| \leq N_{2},\left\|{ }^{C} D^{\alpha} z\right\| \leq N_{3}\right\},
$$

where the positive constants $N_{1}, N_{2}$ and $N_{3}$ are defined by

$$
\begin{aligned}
& N_{1}=d_{2}\left(\left|x_{1}\right|+d_{1}\right) \\
& N_{2}=d_{1}+d_{3} N_{1} \\
& N_{3}=a N_{2}+b_{1} N_{1}+d N_{2} T+K
\end{aligned}
$$

and

$$
\begin{aligned}
a & =\|A\|, \quad b_{1}=\|B\|, \\
b_{2} & =\left\|G^{-1}\right\|, \quad d=\sup \|H(t)\|, \\
a_{1} & =\sup \left\|R_{\alpha}(T)\right\|, \\
a_{2} & =\sup \left\|R_{\alpha, \alpha}(T)\right\|, \\
d_{1} & =a_{1}\left|x_{0}\right|+a_{2} K T^{\alpha} \alpha^{-1}, \\
d_{2} & =a_{2} b_{1} b_{2} \\
d_{3} & =a_{2} b_{1} T^{\alpha} \alpha^{-1} .
\end{aligned}
$$

Using the above notation, we have

$$
\begin{gathered}
\|u(t)\| \leq\left\|B^{*}\right\|\left\|R_{\alpha, \alpha}^{*}(T-s)\right\|\left\|G^{-1}\right\| \\
\quad\left[\left|x_{1}\right|+\left\|R_{\alpha}(T)\right\|\left|x_{0}\right|\right. \\
\quad+\int_{0}^{T}(T-s)^{\alpha-1}\left\|R_{\alpha, \alpha}(T-s)\right\| \\
\left.\quad \times\left\|f\left(s, z(s),{ }^{C} D^{\alpha} z(s), v(s)\right)\right\| \mathrm{d} s\right], \\
\leq a_{2} b_{1} b_{2}\left[x_{1}+a_{1}\left|x_{0}\right|+a_{2} K T^{\alpha} \alpha^{-1}\right], \\
\leq d_{2}\left(\left|x_{1}\right|+d_{1}\right)=N_{1}, \\
\|x(t)\| \leq\left\|R_{\alpha}(T)\right\|\left|x_{0}\right| \\
+\int_{0}^{t}(t-s)^{\alpha-1}\left\|R_{\alpha, \alpha}(t-s)\right\|\|B\|\|u(s)\| \mathrm{d} s \\
+\int_{0}^{t}(t-s)^{\alpha-1}\left\|R_{\alpha, \alpha}(t-s)\right\| \\
\times\left\|^{t}\left(s, z(s),{ }^{C} D^{\alpha} z(s), v(s)\right)\right\| \mathrm{d} s \\
\leq a_{1}\left|x_{0}\right|+a_{2} K T^{\alpha} \alpha^{-1}+a_{2} b_{1} N_{1} T^{\alpha} \alpha^{-1} \\
\leq d_{1}+d_{3} N_{1}=N_{2}
\end{gathered}
$$

and

$$
\left\|{ }^{C} D^{\alpha} x(t)\right\| \leq a N_{2}+b_{1} N_{1}+d N_{2} T+K=N_{3} .
$$

Hence the operator $\Psi$ transforms the set $E$ in $C_{n+m}^{\alpha}(J)$ into itself. It can be easily seen that, for each pair $[z, v] \in E$, we have

$$
\theta\left(\Psi_{2}([z, v], h)\right) \leq \theta\left(S^{*}, h\right) k_{1},
$$

where

$$
S^{*}(T, s)=B^{*} R_{\alpha, \alpha}^{*}(T-s)
$$

and

$$
\begin{aligned}
k_{1}= & \sup _{[z, v] \in E}\left\|G^{-1}\right\|\left\{\left|x_{1}\right|+\left\|R_{\alpha}(T)\right\|\left|x_{0}\right|\right. \\
& +\int_{0}^{T}(T-s)^{\alpha-1}\left\|R_{\alpha, \alpha}(T-s)\right\| \\
& \left.\times\left\|f\left(s, z(s),{ }^{C} D^{\alpha} z(s), v(s)\right)\right\| \mathrm{d} s\right\} .
\end{aligned}
$$


Since the function $S^{*}$ does not depend on the choice of the points in $E$, all the functions $\Psi_{2}([z, v](t))$ have a uniformly bounded modulus of continuity and hence they are equi-continuous. Also all the functions involved in $\Psi_{1}([z, v](t))$ are equicontinuous, since they have uniformly bounded derivatives. Next we have to find an estimate for the modulus of continuity of the functions ${ }^{C} D^{\alpha} \Psi_{1}([z, v](t))$. For that, we have

$$
\begin{aligned}
&\left|{ }^{C} D^{\alpha} \Psi_{1}([z, v](t))-{ }^{C} D^{\alpha} \Psi_{1}([z, v](s))\right| \\
& \leq\left|A \Psi_{1}([z, v](t))-A \Psi_{1}([z, v](s))\right| \\
&+\left|B \Psi_{2}([z, v](t))-B \Psi_{2}([z, v](s))\right| \\
&+ \mid \int_{0}^{t} H(t-s) \Psi_{1}([z, v](\eta)) \mathrm{d} \eta \\
&-\int_{0}^{s} H(s-\eta) \Psi_{1}([z, v](\eta)) \mathrm{d} \eta \mid \\
&+ \mid f\left(t, z(t),{ }^{C} D^{\alpha} z(t), v(t)\right) \\
&-f\left(s, z(s),{ }^{C} D^{\alpha} z(s), v(s)\right) \mid .
\end{aligned}
$$

For the first three terms of the right side of the inequality, we give the upper estimate as $\beta_{0}(|t-s|)$ and $\lim _{h \rightarrow 0} \beta_{0}(h)=0$. Also, it may be chosen independent of the choice of $(z, v)$. For the fourth term, we give the following estimate:

$$
\begin{aligned}
\mid f\left(t, z(t),{ }^{C} D^{\alpha} z(t), v(t)\right)- & f\left(s, z(s),{ }^{C} D^{\alpha} z(s), v(s)\right) \mid \\
\leq & \mid f\left(t, z(t),{ }^{C} D^{\alpha} z(t), v(t)\right) \\
& -f\left(t, z(t),{ }^{C} D^{\alpha} z(s), v(t)\right) \mid \\
+ & \mid f\left(t, z(t),{ }^{C} D^{\alpha} z(s), v(t)\right) \\
& -f\left(s, z(s),{ }^{C} D^{\alpha} z(s), v(s)\right) \mid .
\end{aligned}
$$

For the first term, we have the upper estimate $k\left(\left|{ }^{C} D^{\alpha} z(t)-{ }^{C} D^{\alpha} z(s)\right|\right)$, whereas for the second term, we may find an estimate $\beta_{1}(|t-s|)$ with $\lim _{h \rightarrow 0} \beta_{1}(h)=0$. Hence

$$
\theta\left({ }^{C} D^{\alpha} \Psi_{1}([z, v])(t), h\right) \leq k\left(\theta\left({ }^{C} D^{\alpha} z, h\right)+\beta(h)\right),
$$

where $\beta=\beta_{0}+\beta_{1}$. Therefore, by (5)-8), we conclude that, for any set $E \subset C_{n+m}^{\alpha}(J)$,

$$
\theta_{0}\left(\Psi_{2} E\right)=0, \quad \theta_{0}\left(\Psi_{1} E\right) \leq k \theta_{0}\left({ }^{C} D^{\alpha} E_{2}\right),
$$

where $E_{2}$ is the normal projection of the set $E$ on the space $C_{n}^{\alpha}(J)$. Hence it follows that

$$
\mu(\Psi E) \leq k \mu(E) .
$$

By the Darbo fixed-point theorem, the mapping $T$ has at least one fixed point, therefore there exist functions $u \in C_{m}(J)$ and $x \in C_{n}^{\alpha}(J)$ such that

$$
(x, u)=(z, v)=\left[\Psi_{1}([z, v](t)), \Psi_{2}([z, v](t))\right] .
$$

This shows that $x(t)$ is the solution of (10) for the control $u(t)$ and these functions are the required solution. Hence the system (9) is controllable on $J$.

\section{Neutral fractional integrodifferential systems}

Consider the neutral fractional integrodifferential system governed by the neutral fractional integrodifferential equation with an implicit fractional derivative of the form

$$
\begin{aligned}
{ }^{C} D^{\alpha}[x(t)-g(t, x(t))] & \\
= & A x(t)+\int_{0}^{t} H(t-s) x(s) \mathrm{d} s \\
& +B u(t)+f\left(t, x(t),{ }^{C} D^{\alpha} x(t), u(t)\right), \\
x(0)= & x_{0},
\end{aligned}
$$

where $A, B, H$ and $f$ are as in (4) and the function $g: J \times \mathbb{R}^{n} \rightarrow \mathbb{R}^{n}$ is continuously differentiable. As before, for each fixed point $(z, v) \in C_{n+m}^{\alpha}(J)$, consider the neutral fractional integrodifferential system

$$
\begin{aligned}
{ }^{C} D^{\alpha}[x(t)-g(t, x(t))] \\
=A x(t)+\int_{0}^{t} H(t-s) x(s) \mathrm{d} s \\
\quad+B u(t)+f\left(t, z(t),{ }^{C} D^{\alpha} z(t), v(t)\right),
\end{aligned}
$$

with $x(0)=x_{0}$, and the solution of the system 14 is (Balachandran and Kokila, 2013a)

$$
\begin{aligned}
x(t)= & R_{\alpha}(t) x_{0} \\
& +\int_{0}^{t}(t-s)^{\alpha-1} R_{\alpha, \alpha}(t-s) B u(s) \mathrm{d} s \\
& +\int_{0}^{t}(t-s)^{\alpha-1} R_{\alpha, \alpha}(t-s) \\
& \times f\left(s, z(s),{ }^{C} D^{\alpha} z(s), v(s)\right) \mathrm{d} s \\
& +\frac{1}{\Gamma(1-\alpha)} \int_{0}^{t} \int_{0}^{s}(t-s)^{\alpha-1}(s-\tau)^{-\alpha} \\
& \times R_{\alpha, \alpha}(t-s) g^{\prime}(\tau, x(\tau)) \mathrm{d} \tau \mathrm{d} s .
\end{aligned}
$$

Assume the following additional condition.

The function $g: J \times \mathbb{R}^{n} \rightarrow \mathbb{R}^{n}$ is continuously differentiable and there exists a constant $M>0$ such that

$$
\left\|g^{\prime}(t, x(t))\right\| \leq M \quad \text { for all } \quad t \in J \text { and } x \in \mathbb{R}^{n} .
$$

Theorem 3. If the linear system (2) is controllable on $J$ and the functions $f$ and $g$ satisfy the condition (8) and (16), then the nonlinear system (14) is controllable on J.

Proof. Define the operator $\Phi: C_{n+m}^{\alpha}(J) \rightarrow C_{n+m}^{\alpha}(J)$, as in the work of Balachandran and Kokila (2013b), by

$$
\Phi(z, v)=(x, u),
$$


where

$$
\begin{aligned}
& u(t)=B^{*} R_{\alpha, \alpha}^{*}(T-t) G^{-1}\left[x_{1}-R_{\alpha}(T) x_{0}\right. \\
& -\int_{0}^{T}(T-s)^{\alpha-1} R_{\alpha, \alpha}(T-s) \\
& \times f\left(s, z(s)^{C} D^{\alpha} z(s), v(s)\right) \mathrm{d} s \\
& -\frac{1}{\Gamma(1-\alpha)} \int_{0}^{T} \int_{0}^{s}(T-s)^{\alpha-1}(s-\tau)^{-\alpha} \\
& \left.R_{\alpha, \alpha}(T-s) g^{\prime}(\tau, x(\tau)) \mathrm{d} \tau \mathrm{d} s\right] \\
& x(t)=R_{\alpha}(t) x_{0}+\int_{0}^{t}(t-s)^{\alpha-1} R_{\alpha, \alpha}(t-s) B \\
& \times B^{*} R_{\alpha, \alpha}^{*}(T-s) G^{-1}\left[x_{1}-R_{\alpha}(T) x_{0}\right. \\
& -\int_{0}^{T}(T-\tau)^{\alpha-1} R_{\alpha, \alpha}(T-\tau) \\
& \times f\left(\tau, z(\tau),{ }^{C} D^{\alpha} z(\tau), v(\tau)\right) \mathrm{d} \tau \\
& -\frac{1}{\Gamma(1-\alpha)} \int_{0}^{T} \int_{0}^{s}(T-s)^{\alpha-1}(s-\tau)^{-\alpha} \\
& \left.\times R_{\alpha, \alpha}(t-s) g^{\prime}(\tau, x(\tau)) \mathrm{d} \tau\right] \mathrm{d} s \\
& +\int_{0}^{t}(t-s)^{\alpha-1} R_{\alpha, \alpha}(t-s) \\
& \times f\left(s, z(s),{ }^{C} D^{\alpha} z(s), v(s)\right) \mathrm{d} s \\
& +\frac{1}{\Gamma(1-\alpha)} \int_{0}^{t} \int_{0}^{s}(t-s)^{\alpha-1}(s-\tau)^{-\alpha} \\
& \times R_{\alpha, \alpha}(t-s) g^{\prime}(\tau, x(\tau)) \mathrm{d} \tau \mathrm{d} s .
\end{aligned}
$$

It can be easily verified that $x(T)=x_{1}$ by inserting $t=T$ in (18). We introduce the right-hand sides of (17) and (18) as a pair of operators $\Phi_{2}([z, v](t))$ and $\Phi_{1}([z, v](t))$, respectively, and define the nonlinear operator $\Phi: C_{n+m}^{\alpha}(J) \rightarrow C_{n+m}^{\alpha}(J)$ by

$$
\Phi([z, v](t))=\left[\Phi_{1}([z, v])(t), \Phi_{2}([z, v])(t)\right] .
$$

Obviously, this operator $\Phi$ is continuous, since all the functions involved in the operator are continuous. To prove that $\Phi$ maps the space $C_{n+m}^{\alpha}(J)$ into itself, define a closed convex subset $\mathcal{H}$ by

$$
\mathcal{H}=\left\{[z, v] ;\|v\| \leq M_{1},\|z\| \leq M_{2},\left\|{ }^{C} D^{\alpha} z\right\| \leq M_{3}\right\}
$$

where the positive constants $M_{1}, M_{2}$ and $M_{3}$ are defined by

$$
\begin{aligned}
& M_{1}=a_{2}\left(\left|x_{1}\right|+a_{1}+a_{4}\right), \\
& M_{2}=a_{1}+a_{3} M_{1}+a_{4}, \\
& M_{3}=a M_{2}+\lambda_{1} M_{1}+d M_{2} T+K+a_{5},
\end{aligned}
$$

and

$$
\begin{aligned}
a & =\|A\|, \quad d=\sup \|H(t)\|, \\
\lambda_{1} & =\|B\|, \quad \lambda_{2}=\left\|G^{-1}\right\|, \\
\omega_{1} & =\sup \left\|R_{\alpha}(T)\right\|, \quad \omega_{2}=\sup \left\|R_{\alpha, \alpha}(T)\right\|, \\
a_{1} & =\omega_{1}\left|x_{0}\right|+\omega_{2} K T^{\alpha} \alpha^{-1}, \\
a_{2} & =\omega_{2} \lambda_{1} \lambda_{2}, \quad a_{3}=\omega_{2} \lambda_{1} T^{\alpha} \alpha^{-1}, \\
a_{4} & =\omega_{2} M \Gamma(\alpha) T, \quad a_{5}=\frac{1}{\Gamma(1-\alpha)} T^{1-\alpha} G^{-1} .
\end{aligned}
$$

Using the above, we have

$$
\begin{aligned}
\|u(t)\| \leq & \omega_{2} \lambda_{1} \lambda_{2}\left[\left|x_{1}\right|+\omega_{1}\left|x_{0}\right|+\omega_{2} K T^{\alpha} \alpha^{-1}\right. \\
& \left.+\omega_{2} M \Gamma(\alpha) T\right] \\
\leq & a_{2}\left(\left|x_{1}\right|+a_{1}+a_{4}\right)=M_{1}, \\
\|x(t)\| \leq & \omega_{1}\left|x_{0}\right|+\omega_{2} K T^{\alpha} \alpha^{-1}+\omega_{2} \lambda_{1} M_{1} T^{\alpha} \alpha^{-1} \\
& +\omega_{2} M \Gamma(\alpha) T \\
\leq & a_{1}+a_{3} M_{1}+a_{4}=M_{2},
\end{aligned}
$$

and

$$
\left\|^{C} D^{\alpha} x(t)\right\| \leq a M_{2}+\lambda_{1} M_{1}+d N_{2} T+K+a_{5}=M_{3} .
$$

Hence the operator $\Phi$ transforms $\mathcal{H}$ into itself. It can be easily seen that, for each pair $[z, v] \in \mathcal{H}$, we have

$$
\theta\left(\Phi_{2}([z, v], h)\right) \leq \theta\left(P^{*}, h\right) k_{2},
$$

where

$$
P^{*}(T, s)=B^{*} R_{\alpha, \alpha}^{*}(T-s)
$$

and

$$
\begin{aligned}
k_{2}= & \sup _{[z, v] \in \mathcal{H}}\left\|G^{-1}\right\|\left\{\left|x_{1}\right|+R_{\alpha}(T)\left|x_{0}\right|\right. \\
& +\int_{0}^{T}(T-s)^{\alpha-1}\left\|R_{\alpha, \alpha}(T-s)\right\|\|f\| \mathrm{d} s \\
& +\frac{1}{\Gamma(1-\alpha)} \int_{0}^{T} \int_{0}^{s}(T-s)^{\alpha-1}(s-\tau)^{-\alpha} \\
& \left.\times\left\|R_{\alpha, \alpha}(T-s)\right\|\left\|g^{\prime}(\tau, x(\tau))\right\| \mathrm{d} \tau \mathrm{d} s\right\} .
\end{aligned}
$$

We show that the operators are equicontinuous. Since the function $P^{*}$ does not depend on the choice of the points in $\mathcal{H}$, all the functions $\Phi_{2}([z, v](t))$ have a uniformly bounded modulus of continuity, and hence they are equicontinuous. Also, all the functions used in $\Phi_{1}([z, v](t))$ are equicontinuous, since they have uniformly bounded derivatives. Next we have to find an 
estimate for the modulus of continuity of the functions ${ }^{C} D^{\alpha} \Phi_{1}([z, v](t))$. For that, we have

$$
\begin{aligned}
&\left|{ }^{C} D^{\alpha} \Phi_{1}([z, v](t))-{ }^{C} D^{\alpha} \Phi_{1}([z, v](s))\right| \\
& \leq\left|A \Phi_{1}([z, v])(t)-A \Phi_{1}([z, v])(s)\right| \\
&+\left|B \Phi_{2}([z, v])(t)-B \Phi_{2}([z, v])(s)\right| \\
&+\mid \int_{0}^{t} H(t-s) \Phi_{1}([z, v](\eta)) \mathrm{d} \eta \\
&-\int_{0}^{s} H(s-\eta) \Phi_{1}([z, v](\eta)) \mathrm{d} \eta \mid \\
&+\mid f\left(t, z(t),{ }^{C} D^{\alpha} z(t), v(t)\right) \\
&-f\left(s, z(s),{ }^{C} D^{\alpha} z(s), v(s)\right) \mid \\
&+\left|{ }^{C} D^{\alpha} g(t, x(t))-{ }^{C} D^{\alpha} g(s, x(s))\right| \\
& \leq\left|A \Phi_{1}([z, v])(t)-A \Phi_{1}([z, v](s))\right| \\
&+\mid \int_{0}^{t} H(t-s) \Phi_{1}([z, v](\eta)) \mathrm{d} \eta \\
&-\int_{0}^{s} H(s-\eta) \Phi_{1}([z, v](\eta)) \mathrm{d} \eta \mid \\
&+\left|B \Phi_{2}([z, v](t))-B \Phi_{2}([z, v](s))\right| \\
&+\left|{ }^{C} D^{\alpha} g(t, x(t))-{ }^{C} D^{\alpha} g(s, x(s))\right| \\
&+ \mid f\left(t, z(t),{ }^{C} D^{\alpha} z(t), v(t)\right) \\
&- f\left(t, z(t),{ }^{C} D^{\alpha} z(s), v(t)\right) \mid \\
&+ \mid f\left(t, z(t),{ }^{C} D^{\alpha} z(s), v(t)\right) \\
&- f\left(s, z(s),{ }^{C} D^{\alpha} z(s), v(s)\right) \mid .
\end{aligned}
$$

For the first four terms of the right-hand side of the inequality, we give the upper estimate as $\beta_{0}(|t-s|)$ and the last term by $k\left(\left|{ }^{C} D^{\alpha} z(t)-{ }^{C} D^{\alpha} z(s)\right|\right)+\beta_{1}(|t-s|)$, with $\lim _{h \rightarrow 0} \beta_{i}(h)=0$. Hence $\theta\left({ }^{C} D^{\alpha} \Phi_{1}([z, v])(t), h\right) \leq$ $k\left(\theta\left({ }^{C} D^{\alpha} z, h\right)+\beta(h)\right)$, where $\beta=\beta_{0}+\beta_{1}$. Therefore, by (16) and (5)-(8), we conclude that, for any set $\mathcal{H} \subset$ $C_{n+m}^{\alpha}(J)$,

$$
\theta_{0}\left(\Phi_{2} \mathcal{H}\right)=0, \quad \theta_{0}\left(\Phi_{1} \mathcal{H}\right) \leq k \theta\left({ }^{C} D^{\alpha} \mathcal{H}_{2}\right)
$$

where $\mathcal{H}_{2}$ is the normal projection of the set $\mathcal{H}$ on the space $C_{n}^{\alpha}(J)$. Hence

$$
\mu(\Phi \mathcal{H}) \leq k \mu(\mathcal{H})
$$

By the Darbo fixed-point theorem, the mapping $\Phi$ has at least one fixed point. Therefore, there exist functions $u \in C_{m}(J)$ and $x \in C_{n}^{\alpha}(J)$ such that $\Phi(x, u)=(x, u)$, that is,

$$
u(t)=\Phi_{2}(x, u)(t), \quad x(t)=\Phi_{1}(x, u)(t) .
$$

These functions give the required solution and satisfy $x(T)=x_{1}$. Hence the system (14) is controllable.

\section{Examples}

In this section we give two examples to illustrate the theory developed in the previous sections.

Example 1. Consider the fractional integrodifferential system with an implicit fractional derivative of the form

$$
\begin{aligned}
{ }^{C} D^{\alpha} x(t)= & A x(t)+\int_{0}^{t} H(t-s) x(s) \mathrm{d} s+B u(t) \\
& +f\left(t, x(t),{ }^{C} D^{\alpha} x(t), u(t)\right), t \in J, \\
x(0)= & x_{0},
\end{aligned}
$$

where

$$
\begin{gathered}
A=\left(\begin{array}{ll}
2 & 0 \\
0 & 2
\end{array}\right), \quad B=\left(\begin{array}{ll}
1 & 0 \\
1 & 1
\end{array}\right), \\
H(t-s)=\left(\begin{array}{cc}
\frac{3(t-s)^{-1 / 2}}{\Gamma(1 / 2)} & 0 \\
0 & \frac{3(t-s)^{-1 / 2}}{\Gamma(1 / 2)}
\end{array}\right),
\end{gathered}
$$

$\alpha=1 / 2$ and the nonlinear term $f$ is given by

$$
\begin{aligned}
f\left(t, x(t),{ }^{C} D^{1 / 2} x(t), u(t)\right) & \\
& =\left(\begin{array}{c}
0 \\
t \cos x(t)+\sin \frac{1}{2}\left({ }^{C} D^{1 / 2} x(t)\right)
\end{array}\right) .
\end{aligned}
$$

Here

$$
x(t)=\left(\begin{array}{c}
x_{1}(t) \\
x_{2}(t)
\end{array}\right)
$$

with

$$
x_{1}(t)=x(t), \quad{ }^{C} D^{1 / 4} x_{1}(t)=x_{2}(t) .
$$

First, we consider the homogeneous part of the above system,

$$
\begin{aligned}
& { }^{C} D^{1 / 2} x(t) \\
& =\left(\begin{array}{ll}
2 & 0 \\
0 & 2
\end{array}\right) x(t) \\
& \quad+\int_{0}^{t}\left(\begin{array}{cc}
\frac{3(t-s)^{-1 / 2}}{\Gamma(1 / 2)} & 0 \\
0 & \frac{3(t-s)^{-1 / 2}}{\Gamma(1 / 2)}
\end{array}\right) x(s) \mathrm{d} s .
\end{aligned}
$$

Using the Laplace transform, we find the solution of the system (20) as

$$
x(t)=R_{1 / 2}(t) x(0),
$$

where

$$
R_{1 / 2}(t)=\left(\begin{array}{cc}
Q(t) & 0 \\
0 & Q(t)
\end{array}\right)
$$


Here

$$
Q(t)=\frac{1}{4} E_{1 / 2}\left(t^{1 / 2}\right)+\frac{3}{4} E_{1 / 2}\left(-3 t^{1 / 2}\right)
$$

and $R_{1 / 2}(t)$ is the resolvent matrix which satisfies the following properties:

(i) $R_{1 / 2}(0)=I$,

(ii) ${ }^{C} D^{1 / 2} R_{1 / 2}(t)$

$$
=A R_{1 / 2}(t)+\int_{0}^{t} H(t-s) R_{1 / 2}(s) \mathrm{d} s,
$$

(iii) $\mathcal{L}\left\{R_{1 / 2}(t)\right\}(s)=\int_{0}^{t} e^{-s t} R_{1 / 2}(t) \mathrm{d} t$ $:=s^{-1 / 2}\left(s^{1 / 2} I-A-\mathcal{L}(H)\right)^{-1}$.

Now, taking Laplace transform on (19) and using the property (iii) as well as a simple partial fraction method, we obtain the solution of (19) as (Balachandran and Kokila, 2013a)

$$
\begin{aligned}
& x(t) \\
= & R_{1 / 2}(t)+\int_{0}^{t}(t-s)^{-1 / 2} R_{1 / 2,1 / 2}(t-s) B u(s) \mathrm{d} s,
\end{aligned}
$$

where

$$
\begin{gathered}
R_{1 / 2,1 / 2}(t)=\left(\begin{array}{cc}
L(t) & 0 \\
0 & L(t)
\end{array}\right), \\
L(t)=\frac{1}{4} E_{1 / 2,1 / 2}\left(t^{1 / 2}\right)+\frac{3}{4} E_{1 / 2,1 / 2}\left(-3 t^{1 / 2}\right) .
\end{gathered}
$$

By simple matrix calculation, one can see that the controllability matrix

$$
\begin{aligned}
G= & \int_{0}^{T}(T-s)^{\alpha-1}\left[R_{\alpha, \alpha}(T-s) B\right] \\
& \times\left[B R_{\alpha, \alpha}(T-s)\right]^{*} \mathrm{~d} s \\
= & \int_{0}^{T}(T-s)^{-1 / 2}\left(\begin{array}{cc}
L^{2} & L^{2} \\
L^{2} & 2 L^{2}
\end{array}\right) \mathrm{d} s
\end{aligned}
$$

is positive definite for any $T>0$. Furthermore,

$$
\begin{aligned}
|f(t, x, y, u)-f(t, x, \bar{y}, u)| & =\left\|\sin \frac{y}{2}-\sin \frac{\bar{y}}{2}\right\| \\
& \leq \frac{1}{2}|y-\bar{y}|
\end{aligned}
$$

and there exists $K>0$ such that $|f(t, x, y, u)| \leq K$, so the hypotheses of Theorem 2 are satisfied. Observe that the control defined by

$$
\begin{aligned}
u(t)= & B^{*} R_{\alpha, \alpha}^{*}(T-t) G^{-1}\left[x_{1}-R_{\alpha}(T) x_{0}\right. \\
& -\int_{0}^{T}(T-s)^{\alpha-1} R_{\alpha, \alpha}(T-s) \\
& \left.\times f\left(s, x(s),{ }^{C} D^{\alpha} x(s), u(s)\right) \mathrm{d} s\right]
\end{aligned}
$$

steers the system (19) from $x_{0}$ and $x_{1}$ and hence the fractional system (19) is controllable on $[0, T]$.

Remark 1. It should be noted that, for $\alpha=1$, the fractional system (19) is reduced to integer order Volterra integrodifferential systems with implicit derivative which was studied by Balachandran and Balasubramaniam (1992).

Example 2. Consider the following neutral fractional integrodifferential system:

$$
\begin{aligned}
&{ }^{C} D^{\alpha}[x(t)-g(t, x(t))] \\
&= A x(t)+\int_{0}^{t} H(t-s) x(s) \mathrm{d} s+B u(t) \\
&+f\left(t, x(t),{ }^{C} D^{\alpha} x(t), u(t)\right), \quad t \in J, \\
& x(0)= x_{0},
\end{aligned}
$$

where $A, B, H$ and $f$ are as above, $\alpha=1 / 2$ and $g$ is taken as

$$
g(t, x(t))=\left(\begin{array}{c}
\frac{x_{1}(t)}{1+x_{2}^{2}(t)} \\
\frac{x_{2}(t)}{1+x_{1}^{2}(t)}
\end{array}\right) .
$$

Note that $g$ is differentiable and the derivative is uniformly bounded. Further, the linear system is controllable and $f\left(t, x(t),{ }^{C} D^{1 / 2} x(t), u(t)\right)$ satisfies the hypotheses of Theorem 3, then the non-linear system (23) is controllable on $[0, T]$.

Example 3. Consider the following nonlinear fractional integrodifferential system represented by the matrix fractional integrodifferential equation:

$$
\begin{aligned}
{ }^{C} D^{\alpha} x(t)= & A x(t)+\int_{0}^{t} H(t-s) x(s) \mathrm{d} s+B u(t) \\
& +f\left(t, x(t),{ }^{C} D^{\alpha} x(t), u(t)\right), \quad t \in J, \\
x(0)= & x_{0},
\end{aligned}
$$

where $A, B$ and $H$ are as above, $\alpha=1 / 2$ and $f$ is taken as

$$
f\left(t, x(t),{ }^{C} D^{1 / 2} x(t), u(t)\right)=\left(\begin{array}{c}
0 \\
\sin x(t) \cos u(t)
\end{array}\right),
$$


since the linear system is controllable and the nonlinear function $f\left(t, x(t),{ }^{C} D^{1 / 2} x(t), u(t)\right)$ does not satisfy the condition stated in Theorem 2. However, by Theorem 3.1 of Balachandran et al. (2012c), the nonlinear system (24) is controllable on $[0, T]$.

\section{Acknowledgment}

The authors wish to thank the referees for many helpful suggestions.

\section{References}

Anichini, G., Conti, G. and Zecca, P. (1986). A note on controllability of certain nonlinear system, Note di Mathematica 6(2): 99-111.

Balachandran, K. (1988). Controllability of nonlinear systems with implicit derivatives, IMA Journal of Mathematical Control and Information 5(2): 77-83.

Balachandran, K. and Dauer, J.P. (1987). Controllability of nonlinear systems via fixed point theorems, Journal of $\mathrm{Op}$ timization Theory and Applications 53(3): 345-352.

Balachandran, K. and Balasubramaniam, P. (1992). A note on controllability of nonlinear Volterra integrodifferential systems, Kybernetika 28(4): 284-291.

Balachandran, K. and Balasubramaniam, P. (1994). Controllability of nonlinear neutral Volterra integrodifferential systems, Journal of the Australian Mathematical Society 36(1): 107-116.

Balachandran, K. and Kokila, J. (2012a). On the controllability of fractional dynamical systems, International Journal of Applied Mathematics and Computer Science 22(3): 523-531, DOI: 10.2478/v10006-012-0039-0.

Balachandran, K. and Kokila, J. (2013a). Constrained controllability of fractional dynamical systems, Numerical Functional Analysis and Optimization 34(11): 1187-1205.

Balachandran, K. and Kokila, J. (2013b). Controllability of nonlinear implicit fractional dynamical systems, IMA Journal of Applied Mathematics 79(3): 562-570.

Balachandran, K., Kokila, J. and Trujillo, J.J. (2012b). Relative controllability of fractional dynamical systems with multiple delays in control, Computers and Mathematics with Applications 64(10): 3037-3045.

Balachandran, K., Park, J.Y. and Trujillo, J.J. (2012c). Controllability of nonlinear fractional dynamical systems, Nonlinear Analysis: Theory, Methods and Applications 75(4): 1919-1926.

Balachandran, K., Zhou, Y. and J. Kokila, J. (2012d). Relative controllability of fractional dynamical systems with delays in control, Communications in Nonlinear Science and $\mathrm{Nu}$ merical Simulation 17(9): 3508-3520.

Burton, T.A. (1983). Volterra Integral and Differential Equations, Academic Press, New York, NY.

Caputo, M. (1967). Linear model of dissipation whose Q is almost frequency independent, Part II, Geophysical Journal of Royal Astronomical Society 13(5): 529-539.
Dacka, C. (1980). On the controllability of a class of nonlinear systems, IEEE Transaction on Automatic Control 25(2): 263-266.

Kaczorek, K. (2011). Selected Problems of Fractional Systems Theory, Springer, Berlin.

Kexue, L. and Jigen, P. (2011). Laplace transform and fractional differential equations, Applied Mathematics Letters 24(12): 2019-2013.

Kilbas, A.A., Srivastava, H.M. and Trujillo, J.J. (2006). Theory and Applications of Fractional Differential Equations, Elsevier, Amsterdam.

Klamka, J. (1975a). On the global controllability of perturbed nonlinear systems, IEEE Transactions on Automatic Control AC-20(1): 170-172.

Klamka, J. (1975b). On the local controllability of perturbed nonlinear systems, IEEE Transactions on Automatic Control AC-20(2): 289-291.

Klamka, J. (1975c). Controllability of nonlinear systems with delays in control, IEEE Transactions on Automatic Control AC-20(5): 702-704.

Klamka, J. (1993). Controllability of Dynamical Systems, Kluwer Academic, Dordrecht.

Klamka, J. (1999). Constrained controllability of dynamic systems, International Journal of Applied Mathematics and Computer Science 9(2): 231-244.

Klamka, J. (2000). Schauder's fixed-point theorem in nonlinear controllability problems, Control and Cybernetics 29(1): 153-165.

Klamka, J. (2001). Constrained controllability of semilinear delayed systems, Bulletin of the Polish Academy of Sciences: Technical Sciences 49(3): 505-515.

Klamka, J. (2008). Constrained controllability of semilinear systems with delayed controls, Bulletin of the Polish Academy of Sciences: Technical Sciences 56(4): 333-337.

Klamka, J. (2010). Controllability and minimum energy control problem of fractional discrete time systems, in D. Baleanu, Z.B. Guvenc, and J.A.T. Machado (Eds.), New Trends in Nanotechnology and Fractional Calculus, Springer-Verlag, New York, NY, pp. 503-509.

Miller, K.S. and Ross, B. (1993). An Introduction to the Fractional Calculus and Fractional Differential Equations, Wiley, New York, NY.

Mittal, R.C. and Nigam, R. (2008). Solution of fractional integrodifferential equations by Adomian decomposition method, International Journal of Applied Mathematics and Mechanics 4(2): 87-94.

Oldham, K.B and Spanier, J. (1974). The Fractional Calculus, Academic Press, London.

Olmstead, W.E. and Handelsman, R.A. (1976). Diffusion in a semi-infinite region with nonlinear surface dissipation, SIAM Review 18(2): 275-291.

Podlubny, I. (1999). Fractional Differential Equations, Academic Press, New York, NY. 
Rawashdeh, E.A. (2011). Legendre wavelets method for fractional integrodifferential equations, Applied Mathematical Sciences 5(2): 2467-2474.

Sadovskii, J.B. (1972). Linear compact and condensing operator, Russian Mathematical Surveys 27(1): 85-155.

Sabatier, J., Agarwal, O.P. and Tenreiro Machado, J.A. (Eds.) (2007). Advances in Fractional Calculus: Theoretical Developments and Applications in Physics and Engineering, Springer-Verlag, New York, NY.

Samko, S.G., Kilbas, A.A. and Marichev, O.I. (1993). Fractional Integrals and Derivatives; Theory and Applications, Gordon and Breach, Amsterdam.

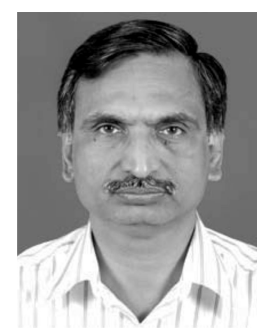

Krishnan Balachandran is a professor in the Department of Mathematics, Bharathiar University, Coimbatore, India. He received the M.Sc. degree in mathematics in 1978 from the University of Madras, Chennai, India. He obtained his M.Phil. and Ph.D. degrees in applied mathematics in 1980 and 1985, respectively, from the same university. In the years 1986-1988, he worked as a lecturer in mathematics at Madras University, P.G. Centre Salem. In 1988, he joined Bharathiar University, Coimbatore, as a reader in mathematics and subsequently was promoted to a professor in 1994. He received the Fulbright Award (1996), the Chandna Award (1999), and the Tamil Nadu Scientists Award (1999) for his research contributions. He has served as a visiting professor at Sophia University (Japan), as well as Pusan National University and Yonsei University (South Korea). He has been a visiting scientist at ICTP, Trieste (Italy), University of Wyoming (USA), Naval Postgraduate School (Monetrey, USA), Hanoi Normal University (Vietnam) and Sungkyunkwan University (Korea). He has published more than 350 technical papers in refereed journals. His major research areas include control theory, abstract integrodifferential equations, stochastic differential equations, fractional differential equations and partial differential equations. He is also a member of the editorial board of the International Journal of Engineering Mathematics and Nonlinear Functional Analysis and Applications.

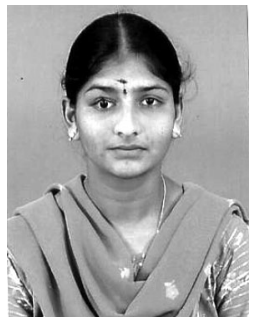

Shanmugam Divya received the B.Sc. degree in mathematics from Bharathiar University, Coimbatore, India, in 2010. She obtained her M.Sc. and M.Phil. degrees in mathematics in 2012 and 2013, respectively, from the same university. Now, she is pursuing her research under the guidance of Prof. K. Balachandran at Bharathiar University. Her research area is the controllability problem of fractional dynamical systems.

Received: 5 November 2013

Revised: 28 February 2014 